\title{
Practical Measurements of Partial Discharges in a Smart Grid Laboratory
}

\author{
Sonia Barrios ${ }^{1}$, Ian Gilbert ${ }^{1}$, Aritz Hurtado ${ }^{1}$, Patrick Mulroy ${ }^{1}$ Iñaki Orue ${ }^{1}$ \\ ${ }^{1}$ Ormazabal Corporate Technology, A.I.E., Spain
}

\begin{abstract}
On-line measurement and monitoring of partial discharges in an MV cable system, including terminations and joints, is a challenging subject because it interacts with other components of the distribution network such as ring main units. The stochastic nature of partial discharges, the different configurations of the network, the external noise and the lack of standard recommendations have consequentially made the quantification of this phenomena more difficult. This paper is an attempt to investigate the behaviour of a theoretical pulse propagation and a real partial discharge in different circuits with almost the same configuration, that can be easily configured in a real Smart Grid laboratory. Several experiments and measurements were performed in order to compare these circuits and to find a factor that represents the influence of different circuit components to use as a reference or calibration to ensure the validity of further measurements.
\end{abstract}

Index Terms-Partial Discharges, measurement, smart grid

\section{INTRODUCTION}

Partial discharges (PDs) appear in a high-voltage energized circuit when the dielectric strength of the insulation is momentarily broken, e.g. air, XLPE cable insulation, SF6, etc. From an electromagnetic point of view they manifest themselves as transient currents pulses at very high frequency ranges, short duration of a few nanoseconds and small magnitude with respect to the circuit power wave.

The indication of partial discharge presence in an electrical network helps us to have a real-time diagnosis of the state of our installation to prevent consumer losses or future failures. Nowadays, partial discharges measurement are categorized in Conventional and No-Conventional approaches [1], the latter including on-line measurement that is outside the scope of IEC 60270 international standard.

Different techniques are used, depending on the manufacturers and service providers making it difficult to compare quantitative measurements e.g. [2-5]. In addition to this problem, the fact of having different distribution network topologies and the difficulty of performing repeated tests under the same conditions in an energized installation make it complicated to standardize and characterize properly the partial discharge behaviour and quantification in a complex environment.

Studies have shown [6] that the signal energy of a detected PD signal decreases for increasing distance between the PD origin and the measuring point due to attenuation in cables. Most signal loss is due to substations, because the propagation coefficient of this component is unknown and it acts as a complex impedance formed by the combination of the influence of switchgear, transformer, MV cables, and other components such as line reactors.

In order to analyse these issues, practical experiments and measurements are performed in a Medium Voltage (MV) distribution demonstration and experimentation network. The PD monitoring system used for these measurements includes capacitive coupling units, installed directly into the T-junction cable end-plug within the switchgear enclosure [7]. Ormazabal's Smart Grid Laboratory, called UDEX, provides the capability of developing and testing new technologies, products and services in a highly configurable medium voltage network independent from the utility grid in a safe and controlled environment [8]. The laboratory single-line diagram with different line topologies that are possible to interconnect is shown in Figure 1.

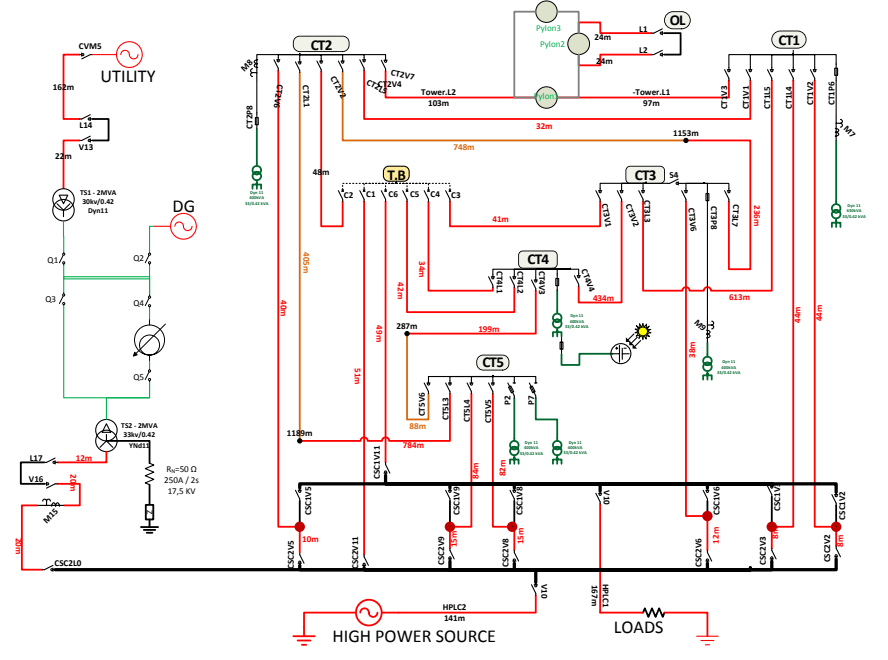

Fig. 1. Laboratory line topologies.

It has a Test-Bay (TB) connected to five different permanent substations by underground cables. It can be powered by four different power sources and the voltage can be controlled up to $36 \mathrm{kV}$.

\section{EXPERIMENTAL SETUP}

Nowadays, the main reason for PD failures is poor workmanship in joints and terminations, and not the cable itself [9][10]. Considering this, a real PD source will be introduced in the TB, generating a floating potential discharge type (Fig. 2). 


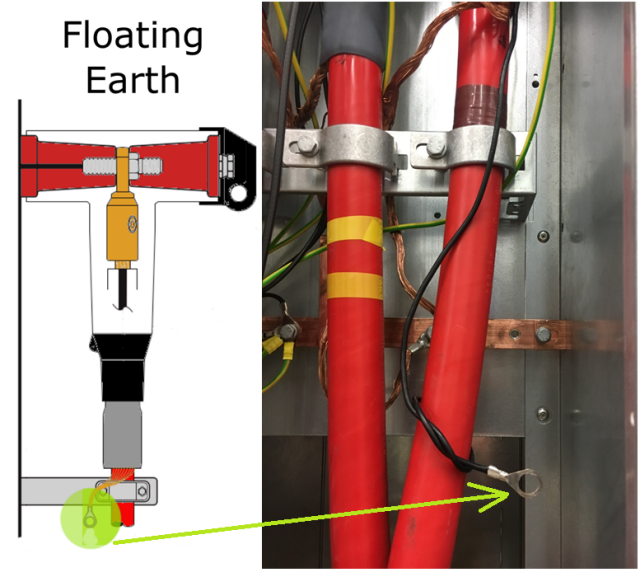

Fig. 2. Floating earth in the switchgear cubicle.

This is a common installation error when the conductor earth is not properly connected in the cubicle.

Taking advantage of the flexibility in accessing and configuring UDEX, four circuits were used to analyse partial discharge signal behaviour, in particular the relation between signal attenuation and circuit components (Fig. 3).

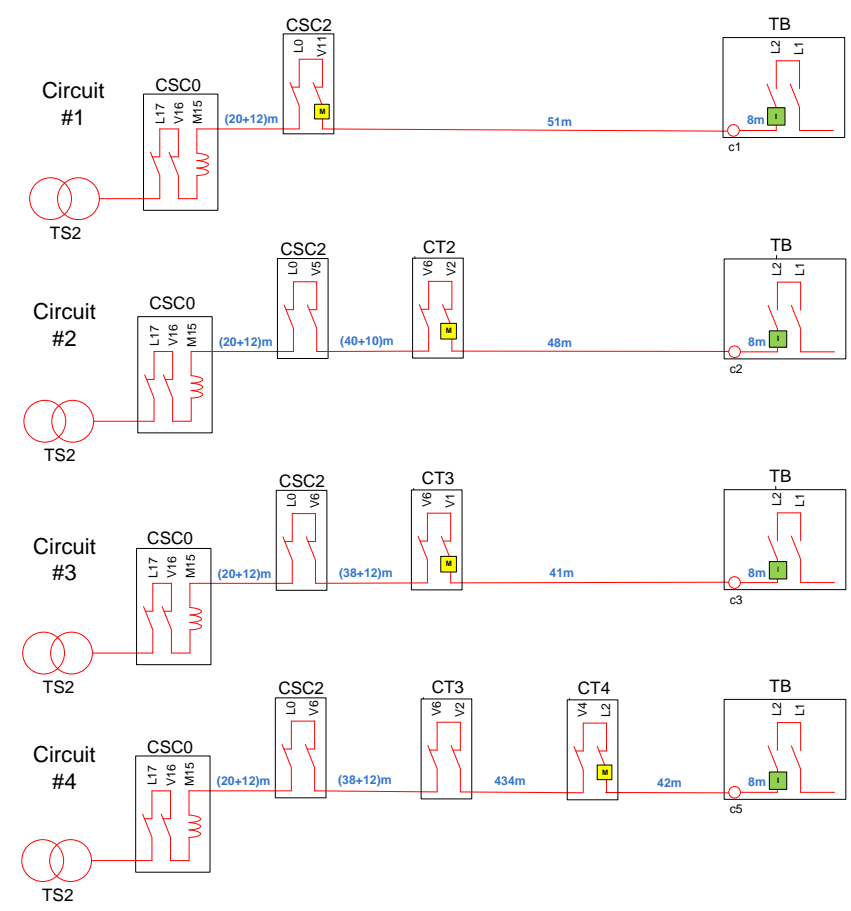

Fig. 3. Different circuits used in these experiments.

Those circuits were chosen in order to have almost the same cable length between PD source or signal injection at the TB (green point) and the measure at the closest switchgear cubicle ; CSC2, CT2, CT3, CT4 respectively (yellow point). As PD pulses will be attenuated, dispersed and reflected while propagating in the cable system, the effect of other circuit components upstream from the measuring point will be analysed. All circuits have the same XLPE cable without any joint.

Before experimenting with the real PD source, a HF signal is injected in only phase 1 of each circuit to study a theoretical PD signal propagation (Fig. 4).

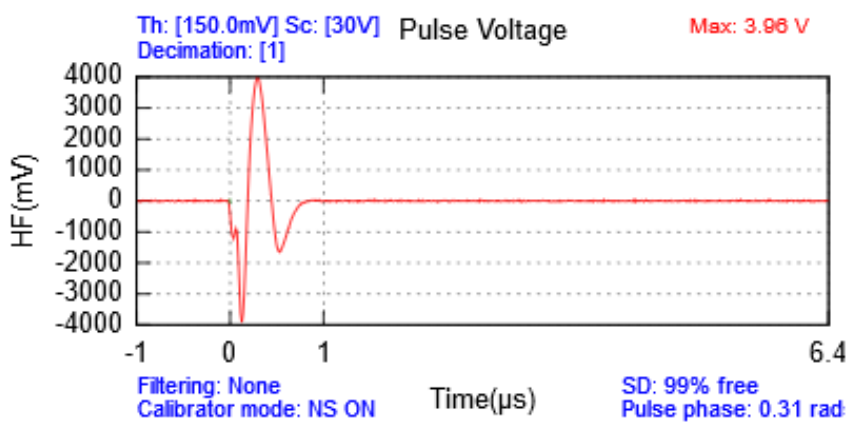

Fig. 4. Reference signal injected through capacitive sensor.

\section{RESULTS \& DISCUSSION}

\section{A. Off-line Measurements}

In order to see the behaviour of the theoretical PD signal that propagates only in the cable, the first test is without energizing the circuit, i.e. the switchgear closest to the TB is not connected upstream. The recorded voltage and the waveform obtained at each measurement point of each circuit is shown in Figure 5.
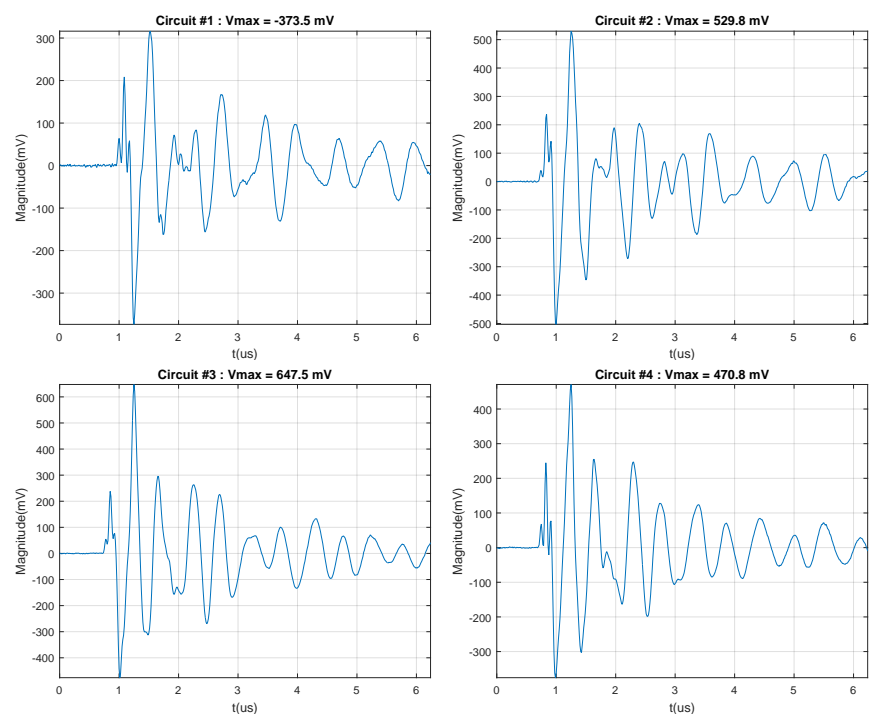

Fig. 5. Theoretical PD signal. Circuit Off-Line results.

Firstly, we have seen the difference between the injection signal waveform and the measurement signal waveform where we have the principal injected signal attenuated and the reflection through the cable. It is seen that the shortest distance between injection and measurement is the CIRCUIT\#3 (49m) 
and it has less attenuation $(647.5 \mathrm{mV})$ than CIRCUIT\#1 $(373.5 \mathrm{mV})$, the longest one $(59 \mathrm{~m})$. Otherwise, it was expected to measure the same attenuation in CIRCUIT\#3 and CIRCUIT\#4 as they only slightly differ by $1 \mathrm{~m}$ but there is a $176 \mathrm{mV}$ difference between them. Slight differences in the impedance mismatch between the cable and switchgear in both circuit setups and the magnitudes of resulting reflections might explain this difference in magnitude observed.

\section{B. On-line Measurements}

1) Theoretical PD Signal: The second experiment is to energize each circuit at $10 \mathrm{kV}$ and inject the theoretical signal. In this case we will have the influence of the different components of the circuit and the total cable system length.
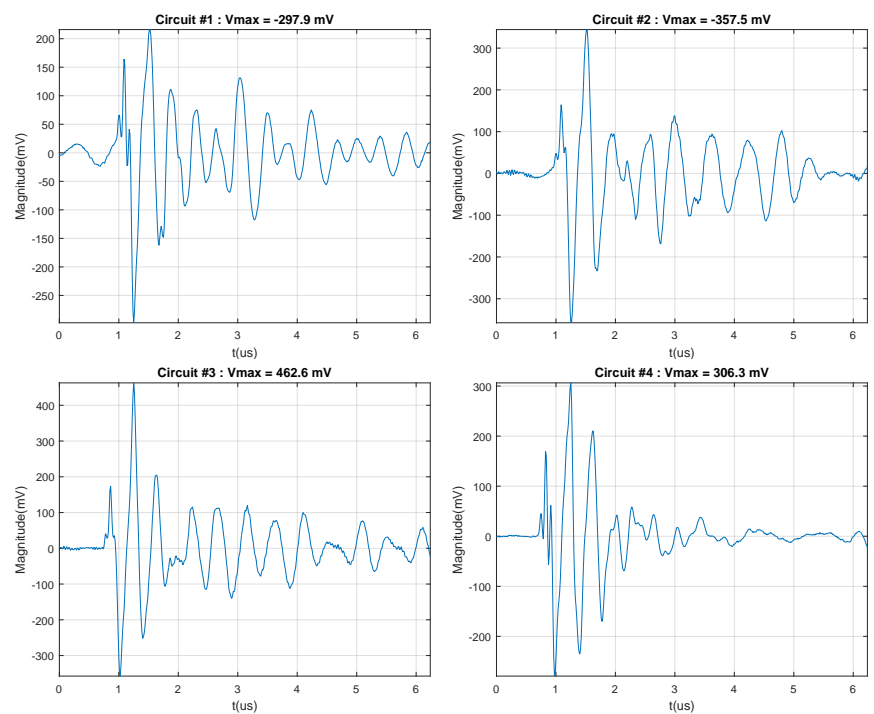

Fig. 6. Theoretical PD signal. Circuit On-Line results.

In this case, as shown in Figure 6, we have almost the same signal waveform as in the Off-line case, except for the CIRCUIT\#4 that has the longest cable system length $(566 \mathrm{~m})$, where we see less reflected pulses, because it takes more time to reach the measuring point again.

We also have more signal attenuation due the components upstream from the measuring point. Otherwise, it was expected to measure the same attenuation in CIRCUIT\#3 and CIRCUIT\#2 as they only differ by $7 \mathrm{~m}$ and they have the same circuits components, but there is a difference of 105 $\mathrm{mV}$ between them and we found different pulse polarity.

The unexpected magnitude results could be because in substations, the earth screen of the XLPE cable is electrically connected to the enclosure, which makes the pulses propagate also into earths connection, making this differences in magnitudes and polarity. Hence, a more detailed study of this issue has to be made and others sensor, e.g. a High frequency current Transformer (HFCT), has to be used to complement this measurements.

A test was made exchanging injection point with measurement point and vice versa, we have obtained
TABLE I

COMPARISON BETWEEN TWO PD MEASUREMENTS POINTS

\begin{tabular}{|c||c|}
\hline Measurement near to PD source & Measurement far from PD source \\
\hline $1.98 \mathrm{~V}$ & $266 \mathrm{mV}$ \\
\hline $3.76 \mathrm{~V}$ & $413 \mathrm{mV}$ \\
\hline $2.48 \mathrm{~V}$ & $242 \mathrm{mV}$ \\
\hline $2.24 \mathrm{~V}$ & $333 \mathrm{mV}$ \\
\hline
\end{tabular}

the same results. This was striking because having the measuring point in the TB, a line-end, we were expecting to measure a higher signal magnitude due to contributions from the closer signal reflections. It may be that the measuring point is too close and as such the reflections do not contribute.

2) Real PD Signal: PD source was located at phase 1 in the TB cubicle and all circuits ware energized at $10 \mathrm{kV}$.

The results shown in Figure 7 are measuring the signal in $\mathrm{TB}$, near to the PD source, where we have less attenuation and which was made to compare with the results at the switchgear cubicle as shown in Table I. This highlights the important signal attenuation and the importance of measure equipment location in the circuit to determinate the PD severity.
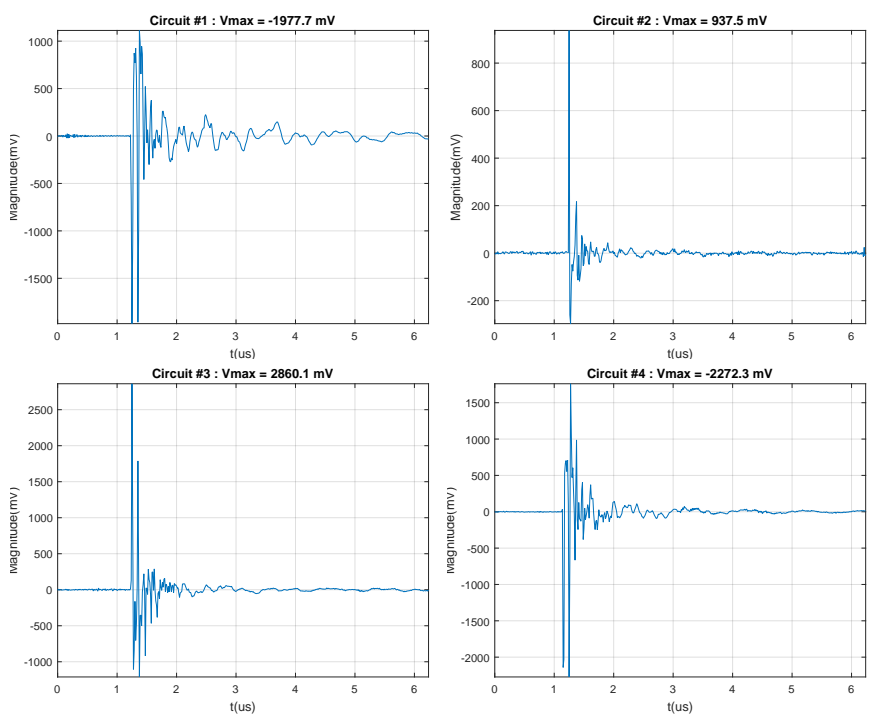

Fig. 7. Real PD signal. Circuit On-Line results.

It must be noted that the magnitude of the signal is not constant and varies significantly with time. The magnitude results depend therefore on the time we registered these values. In this case, the pulse distribution has to be measured as a function of the phase angle of the voltage wave to ensure that the pattern thus obtained correspond to a PD source and not to external noise.

The signal waveform in this case clearly shows the PD pulse with less reflected pulses, this is because as the PD signal is not constant the pulse loses energy before reaching the measuring point again. 

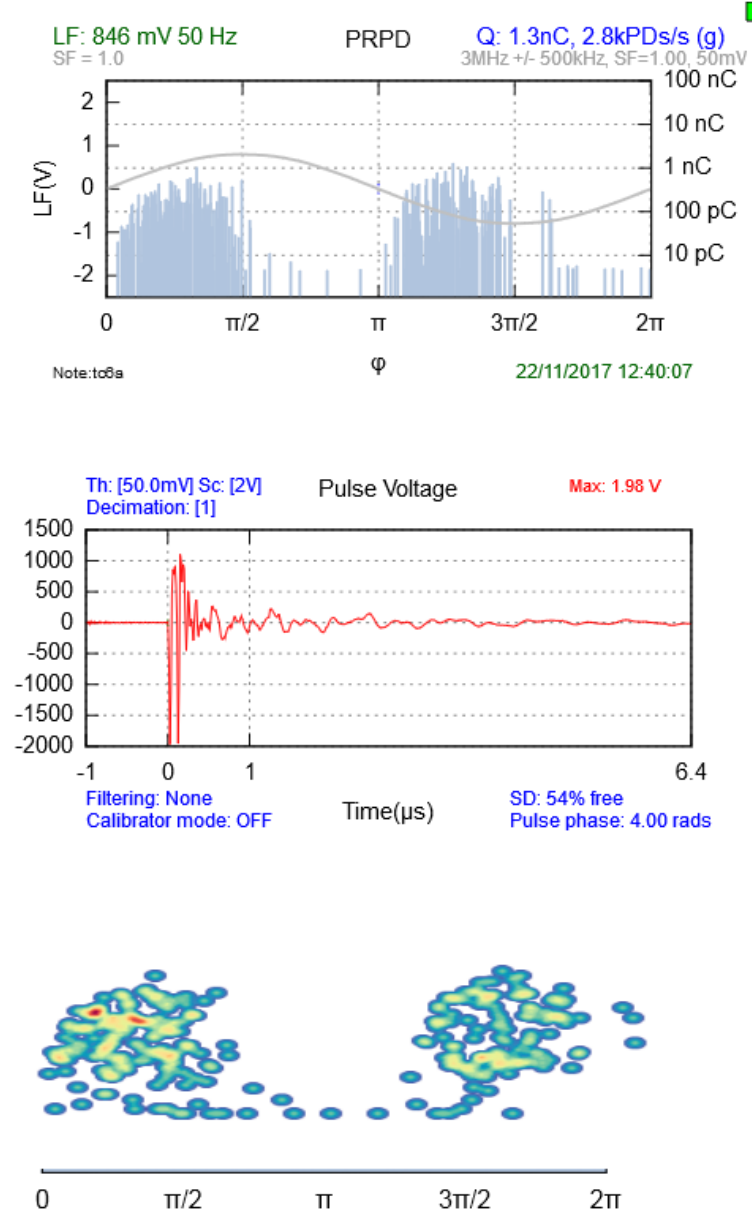
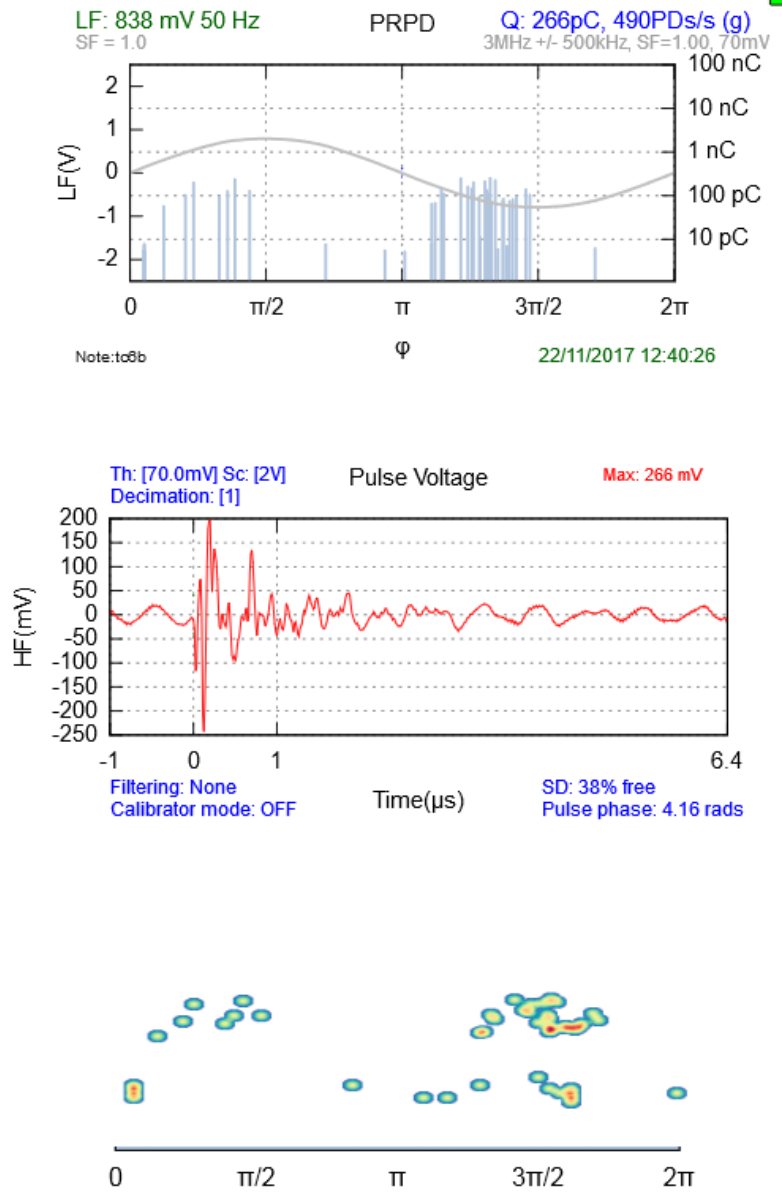

Fig. 8. PD monitoring system interface.

In Figure 8, results from the PD monitoring system interface are shown to compare the near and the far measuring point of the PD in CIRCUIT \#1. At the top of this figure, we can see the low frequency power wave superimposed with the high frequency PD pulses, here it is clearly seen that these pulses are not constant and they do not have the same amplitude. In the middle, we have the PD pulse waveform, that was discussed previously. Finally, at the bottom, we can also see the PD pattern that indicates a typical floating effect. Even if the PD magnitude measured at the far measuring point is lower, with this pattern we can be certain that we have a PD source in our installation and action will have to be taken.

\section{CONCLUSION}

Several circuits were configured at Ormazabal MV Smart Grid (UDEX) and used to analyse typical partial discharge signal behaviour, in particular the relation between signal attenuation and circuit components.

The PD On-line monitoring system is an important tool to know the network insulation state. We highlight in this paper the difficulties and challenges to quantify and measure PD activity even when considering the same circuit configuration.
Analysing the different circuits, even if it could be assumed that the amplitude of signal injected at one end should decrease as a function of measuring distance and the different components in the circuit. These expected results were not experimentally observed analysing only the pulse magnitude. Therefore, others features of the pulse and the circuit must be taken into account as was discussed, and measurements with other equipments must be also performed to compare the results.

The results from this and future work that have been carried out in a real distribution network are beneficial to the operator in planning the measurement strategy in a network. With previous knowledge of network topology, it would be possible to install and measure at specific points in the network knowing the influence on measurements, and in the interpretation of measurement results.

\section{ACKNOWLEDGMENT}

This project has received funding from the European Unions Horizon 2020 research and innovation programme under the Marie Sklodowska-Curie grant agreement No 676042 


\section{REFERENCES}

[1] "IEC TS 62478:2016 ; High voltage test techniques - Measurement of partial discharges by electromagnetic and acoustic methods," International Electrotechnical Commission, Geneva, CH, Technical Specification, 2016.

[2] X. Gu, Y. Xu, R. Xia, S. Meng, and Y. Wang, "On-line calibration of partial discharge monitoring for power cable by HFCT method," in 2016 IEEE Electrical Insulation Conference (EIC), Jun. 2016, pp. 40-44.

[3] Z. Berler, I. Blokhintsev, A. Golubev, G. Paoletti, V. Rashkes, and A. Romashkov, "Practical experience in on-line partial discharge measurements of MV switchgear systems," in Conference Record of the 2000 IEEE International Symposium on Electrical Insulation (Cat. No.00CH37075), 2000, pp. 382-385.

[4] M. Shafiq, G. A. Hussain, L. Ktt, N. I. Elkalashy, and M. Lehtonen, "Partial discharge diagnostic system for smart distribution networks using directionally calibrated induction sensors," Electric Power Systems Research, vol. 119, pp. 447-461, Feb. 2015.

[5] L. Renforth, A. Burgess, M. Foxall, and T. Raczy, "On-line partial discharge (OLPD) insulation condition monitoring of complete high voltage (HV) networks in the oil amp; gas industry," in 2014 IEEE Petroleum and Chemical Industry Conference - Brasil (PCIC Brasil), Aug. 2014, pp. 14-23.

[6] P. Wagenaars, "Integration of online partial discharge monitoring and defect location in medium-voltage cable networks," phdthesis, Technische Universiteit Eindhoven, Eindhoven, 2010.

[7] P. Mulroy, I. Gilbert, and A. Hurtado, "Continuous online monitoring of PD activity in the medium voltage distribution network," in 22nd International Conference and Exhibition on Electricity Distribution (CIRED 2013), June 2013, pp. 1-4.

[8] I. Gilbert, P. Mulroy, A. Hurtado, A. Nabil, and I. Orue, "Practical Experience of a Partial Discharge Monitoring Application on an Experimentation MV Distribution Network," in 23rd International Conference on Electricity Distribution, 2015.

[9] O. Bergius, "Implementation of On-line Partial Discharge Measurements in Medium Voltage Cable Network," mastersthesis, 2012. [Online]. Available: https://dspace.cc.tut.fi/dpub/handle/123456789/20964

[10] L. Zhong, Y. Xu, G. Chen, A. E. Davies, Z. Richardson, and S. G. Swingler, "Use of capacitive couplers for partial discharge measurements in power cables and joints," in Proceedings of the 2001 IEEE 7th International Conference on Solid Dielectrics, 2001. ICSD '01, 2001, pp. 412-415. 